\title{
Severe \& Moderate BPH Symptoms in Mid-Aged Men Improve with Isoflavonoid-Equol Treatment: Pilot Intervention Study
}

\author{
Edwin D. Lephart ${ }^{1,2}$ \\ ${ }^{1}$ The Department of Physiology and Developmental Biology, Brigham Young University, Provo, USA \\ ${ }^{2}$ The Neuroscience Center, Brigham Young University, Provo, USA \\ Email: Edwin_Lephart@byu.edu
}

Received November 7, 2012; revised December 16, 2012; accepted December 28, 2012

\begin{abstract}
Benign prostatic hyperplasia (BPH) is the pathological cellular progression of glandular proliferation associated with aging. Current available treatment options for BPH have limitations and various adverse effects. Equol is a polyphenolic/isoflavonoid molecule derived from intestinal metabolism, dairy and dietary plant sources. It has the unique characteristic to bind specifically $5 \alpha$-dihydrotestosterone ( $5 \alpha$-DHT) by sequestering $5 \alpha$-DHT from the androgen receptor, thus decreasing androgen hormone actions to improve prostate health by acting as a selective androgen modulator (SAM). It also has affinity for estrogen related receptor gamma (ERR- $\gamma)$ and estrogen receptor beta (ER- $\beta$ ) within the prostate that is known to improve male health via selective estrogen receptor modulatory (SERM) activities to decrease inflammation, cellular proliferation and carcinogenesis. We investigated the possible clinical efficacy of equol on the symptoms associated with benign prostatic hyperplasia (BPH) in this study. Materials and Methods: We performed a pilot intervention study evaluating the effects of low dose oral equol supplement (6 mg, twice a day with meals) for 4 weeks in a total of 18 men (49 - 60 years old) with moderate or severe BPH. Subjects included in the study: gave informed consent, underwent a physical examination and verified their BPH symptoms as measured by the International Prostate Symptom Scores (IPSS) and then were assigned to the moderate or severe BPH groups based upon their total IPSS index. All adverse events were reported. The primary efficacy measure was the IPSS parameters comparing baseline to 2 and 4 week IPSS indices. Blood samples were collected at the baseline and 4th week visits that served as secondary efficacy parameters that included testosterone, $5 \alpha$-DHT and general blood chemistries along with cardiac and hepatic function panels. Results: Low dose equol positively improved moderate to severe BPH symptoms according to the IPSS indices. In moderately symptomatic men $(n=10) 5$ out of 7 of the IPSS parameters significantly improved by 4 weeks of equol treatment. In severely symptomatic men $(n=8)$ all 7 of the IPSS parameters significantly improved with 4 weeks of equol treatment. There were no significant changes in androgen levels, general blood chemistries or cardiac and hepatic function parameters. Although, $5 \alpha$-DHT levels declined by $21 \%$ in severely symptomatic men (from baseline vs 4 week values). Conclusion: These findings suggest that equol may provide a well tolerated and rapid beneficial therapy for BPH that can be used alone or in combination with current pharmaceutical therapies. The beneficial clinical efficacy of equol observed in this study may be due to the multiple positive biological actions that are not present in current pharmaceutical treatments.
\end{abstract}

Keywords: Isoflavonoid; Equol; BPH; Androgens; SERM

\section{Introduction}

Benign prostatic hyperplasia (BPH) is the pathological progression of epithelial and stromal proliferation in the prostate gland and the most common benign neoplasm in American men [1]. Prostatic histological changes begin in the third to fourth decade of life and clinically in the fifth decade of life [1]. These changes are mediated primarily by $5 \alpha$-dihydrotestosterone ( $5 \alpha$-DHT) levels within the prostate $[1,2]$ but estrogen hormonal actions via estrogen related receptor (ERR) gamma $(\gamma)$ and estrogen receptor (ER) subtypes presumably play roles in $\mathrm{BPH}$ status $[3,4]$. There appears to be a linear increase in BPH incidence with increasing age in men over 60 years old $[1,2,5]$. It is estimated that almost 7 million Caucasian men in the United States (in 2000) have BPH and nearly 8 million physician office visits were made to diagnose BPH [1]. The estimated costs of BPH (in 2000) exceeded $\$ 1$ billion and approximately 1 in 5 men with BPH had a clinical event, such as prostatectomies, within 1 year of initiating treatment for $\mathrm{BPH}[1,6]$. The symptoms of 
BPH, such as nocturia (the most common, causing poor quality of sleep), incomplete emptying, urinary hesitancy, weak stream, frequency, and urgency can have a significant negative impact on the quality of life $[1,2,6]$.

Pharmacological management of BPH include selective and non-selective $\alpha$-blockers (fast acting-hours to days) and $5 \alpha$-reductase inhibitors (5ARI; relatively slow acting-days to months) for men with symptomatic BPH [2]. In some cases, combination therapy of $\alpha$-blockers and 5ARIs are employed due to different and complementary modes of action that potentially maximize treatment response or efficacy [2]. International prostate symptom scores (IPSS) improve with both modes of pharmacological treatment. However, the most common side effects of $\alpha$-blockers include: reduced ejaculatory volumes, dizziness, diarrhea, orthostatic hypotension, headache, nasopharyngitis and nasal congestion [2,7]. For 5ARIs the most common adverse events are sexual dysfunction including, reduced libido, erectile dysfunction and to a lesser extent ejaculation disorders [2]. Also gynecomastia can occur in $1 \%-2 \%$ of patients with 5 ARIs [2]. Finally, the goal of therapy is to reduce or alleviate $\mathrm{BPH}$ symptoms, to prevent complications, and to minimize adverse effects of treatments [8].

Interest in complementary and alternative medicines (CAM) for BPH increased after epidemiological and traditional hypothesis-driven scientific studies showed a lower incidence of $\mathrm{BPH}$ and prostate cancer in Asians compared to persons in Western countries [9-12]. This caused a paradigm-shift to explore other known and/or previously unknown "natural" treatments for BPH that have reduced side effects. For example, it has been known for centuries that particular plants or plant-derived compounds have prostate health benefits. Several CAM agents to treat BPH include: saw palmetto, pygeum, stinging nettle, lycopene, pumpkin seeds, $\beta$-sitosterol, green tea and vitamins $\mathrm{C}$ and $\mathrm{E}$. These agents can be used as either single or combination therapies [13]. Of these CAM agents, saw palmetto is one of the most common treatments for BPH symptoms, being in some cases as effective as 5ARIs (but not as effective as other pharmaceuticals) with rare side effects $(<3 \%)[14,15]$.

Other CAM agents include polyphenolic compounds such as resveratrol or the isoflavonoids [16,17]. Increased research attention directed toward the isoflavonoids in the last 20 years where genistein was first thought to be responsible for improved prostate health $[9,11]$, however, since the equol hypothesis was proposed in the late 1990s there has been increased focus on this isoflavonoid molecule [18].

Equol is a polyphenolic or isoflavonoid molecule derived from intestinal metabolism, dairy and dietary plant sources [18-21]. For example, it is well established in human populations approximately $30 \%$ to $50 \%$ of individuals can produce equol at relatively low concentrations compared to animals [18,20]. However, recent evidence suggests equol is present in plant products such as beans, cabbage and lettuces [21]. More recent research has shows that equol concentrations in low-soy-consuming U.S. populations reflect equol intakes from mammalian (cow) milk sources [20] that can be as high as 1.5 $\mathrm{mg} / \mathrm{kg}$ of cow's milk [22]. Therefore, humans are exposed to this polyphenolic compound from different plant and food sources regardless of age, gender or geographical location with scientific data to support a consumption/exposure record that appears to be safe [23]. Also, equol has the unique characteristic to bind specifically $5 \alpha$-DHT (to decrease negative androgen impact in the prostate) by sequestering $5 \alpha$-DHT from the androgen receptor (AR), thus altering growth and physiological hormone responses regulated by $5 \alpha$-DHT $[19,24,25]$. It also has affinity for ERR- $\gamma$ and ER- $\beta$ (to increase positive estrogen-like influences in the prostate) $[3,4,25,26]$ and can be found as R-equol and S-equol isomers [18,25]. Equol is currently used in skin treatments and has many positive influences on dermal gene and protein expression (e.g., anti-aging, anti-inflammatory and anti-oxidant properties) [27], but a recent proposal suggests equol may have beneficial applications for improvement in prostate health [25].

\section{Aim of the Study}

The purpose of this pilot intervention study was to determine whether short-term (4 week) oral administration of equol (6 mg twice per day) would improve BPH in mid-aged Caucasian men with moderate to severe symptoms as indexed by the IPSS.

In brief, the summary of blood chemistries displayed no significant changes in: general, cardiac or hepatic function panels while equol levels increased with dosing. In moderately symptomatic men $(n=10) 5$ out of 7 of the IPSS parameters significantly improved by 4 weeks of equol treatment. In severely symptomatic men $(n=8)$ all 7 of IPSS parameters significantly improved with 4 weeks of equol treatment. The present results demonstrate promise for equol at a low effective dose of $6 \mathrm{mg}$ twice per day to improve BPH symptoms in mid-aged men.

\section{Materials and Methods}

We performed a pilot intervention study to determine the safety and efficacy of short-term (4 week) low dose of equol for BPH symptoms. Community dwelling men 49 to 60 years old with moderate to severe BPH symptoms according to the IPSS total symptom index were recruited. 


\subsection{Participants/Protocol (Inclusion/Exclusion Criteria, Surveys \& Adverse Events)}

Subjects were recruited by media advertisements. The clinic site, protocol, etc. was approved by the institutional review board (IntegReview) to perform this pilot study. After providing informed consent Caucasian subjects underwent initial evaluation, including confirmation of their BPH symptoms (moderate $=8-19$ or severe $=$ 20 - 35) by completing the IPSS index, medical history and physical examination. Subjects were included and assigned to moderate or severe BPH groups based upon the total IPSS index that were in good health, having normal physical examination values and no history of prostate cancer. Exclusion criteria included routine high dosage of: anti-inflammatory, immunosuppressive, antihypertensive or anti-histamine medications, insulin, antibiotics, or other topical treatments, diabetes (type I and II), thyroid conditions, chronic asthma or immunological disorders. Subjects should not have taken within the last 8 weeks selective and non-selective $\alpha$-blockers and/or 5ARIs, or natural products/supplements such as green tea, soy or saw palmetto, etc. Subject age and body mass index information, attrition, adverse events and study start/ stop dates are displayed in Table 1. Also, pre-surveys (before treatment was administered) covering information concerning body weight (recent weight gain/loss), physical activity routines, dietary/eating patterns, work shift schedule, and sleeping patterns were recorded.

\subsection{Interventions, Primary and Secondary Endpoints}

This study consisted of a 4-week treatment period; baseline IPSS index values were recorded (see above). Each subject underwent blood sample collection prior to taking the oral equol supplement (6 mg, propriety R/S blend) in the clinic and approximately $30-45$ minutes after taking the equol supplement for visit 1 and visit 3 (which was the $4^{\text {th }}$ week of equol treatment or the end of the study). Subjects received a two week supply of the equol supplement and were instructed to take 1 oral supplement twice per day with food (at breakfast and dinner). Subjects were asked to record in a daily diary: 1) dietary in-

Table 1. Subject demographics and characteristics.

\begin{tabular}{ccc}
\hline & $\begin{array}{c}\text { Moderately } \\
\text { Symptomatic } \mathrm{n}=10\end{array}$ & $\begin{array}{c}\text { Severely } \\
\text { Symptomatic } \mathrm{n}=8\end{array}$ \\
\hline AGE & $55.8 \pm 1.1$ & $58.0 \pm 1.0$ \\
Body Mass Index & $27.9 \pm 0.6$ & $27.2 \pm 1.6$ \\
\hline
\end{tabular}

Attrition: A total of 20 subjects were enrolled and 18 subjects completed the study. Two subjects discontinued study participation after the first baseline visit due to (adverse events): 1) motor-vehicle accident and 2) GI tract disorder, both unrelated to the equol dosing or study procedures. Study start date: $13^{\text {th }}$ April, stop date $-16^{\text {th }}$ June, $2011 ; n=$ number of subjects. take food; 2) physical activity pattern; 3) time of daily equol doses and 4) document daytime and nighttime voiding patterns. This protocol was repeated after two weeks on the equol treatment (visit 2) and then up to 4 weeks on the equol treatment (visit 3). IPSS scores were adjusted for 2 week interval indexing and the blood samples (serum) were analyzed for clinical chemistries (albumin, sex hormone binding globulin, total protein/bilirubin, alkaline phosphatase, ALT, AST, testosterone and $5 \alpha$-DHT levels) from baseline (visit 1) and week 4 (visit 3) time points at Utah Valley Regional Medical Center, Provo, Utah using standard clinical laboratory methods/techniques. Equol levels were quantified by GCMS.

\subsection{Statistical Analysis}

A mixed model (continuous-repeated variable within subject) was used to test baseline vs 2 week or 4 week IPSS values for all recorded parameters $(\mathrm{p}<0.05)$. For the quantified blood chemistries baseline vs 4 week values were tested by ANOVA followed by pairwise comparisons $(\mathrm{p}<0.05)$. Both analyses used Statistical Analysis Software (SAS)-Analytic Pro-Cary, NC, USA. All data were expressed as the mean \pm SEM.

\section{Results}

Standard blood chemistry parameters and changes in equol levels with dosing are displayed in Table 2. There were no significant changes within or across the moderately symptomatic BPH or severely symptomatic groups for: albumin, sex hormone binding globulin, total protein/bilirubin, alkaline phosphatase, ALT \& AST when baseline values were compared to 4 week levels for these parameters (Table 2). For equol levels/profiles within and across time collection intervals, there were no significant differences between the moderately vs. the severely symptomatic groups (Table 2).

Testosterone and $5 \alpha$-DHT levels are displayed in Figure 1. From baseline to termination of the study at 4 weeks there were no significant changes in testosterone or $5 \alpha$-DHT levels in the moderately symptomatic or severely symptomatic groups. Notably, in the severely symptomatic group $5 \alpha$-DHT levels decreased from 429 $\mathrm{pg} / \mathrm{ml}$ to $340 \mathrm{pg} / \mathrm{ml}$ but this reduction did not reach significance $(p<0.07)$. All androgen values were within normal range.

IPSS indices (for all 7 parameters) as influenced by the equol treatments in the moderately vs the severely symptomatic groups are shown in Figures 2(a) and (b). In the moderately symptomatic group for the IPSS parameters, 5 out of 7 indicators significantly improved by 4 weeks of equol treatment. Only urgency and weak stream did not significantly improve (Figure 2(a)). In the severely symptomatic group all 7 of these IPSS indicators 
Table 2. Blood chemistry parameters and equol Levels in moderately and severely symptomatic men with BPH over the 4 week treatment interval.

\begin{tabular}{ccccc}
\hline & \multicolumn{2}{c}{ Moderately Symptomatic $(\mathbf{n}=\mathbf{1 0})$} & \multicolumn{2}{c}{ Severely Symptomatic $(\mathbf{n}=\mathbf{8})$} \\
\cline { 2 - 5 } Albumin g/dL (3.3 - 4.8) & Baseline & Week 4 & Baseline & Week 4 \\
\hline Sex-Hormone Binding Globulin nMol/L (13 - 57) & $3.78+0.09$ & $3.63+0.11$ & $4.33+0.08$ & $4.24+0.11$ \\
& $33.5+4.5$ & $33.4+4.8$ & $30.9+6.1$ & $26.6+5.3$ \\
Total Protein g/dL (6.0 - 8.4) & Cardiac/Hepatic Function Panel & & $6.8+0.1$ \\
Total Bilirubin mg/dL (0.2 - 1.3) & $6.6+0.2$ & $6.5+0.2$ & $6.9+0.1$ & $0.59+0.13$ \\
Alkaline Phosphatase U/L (40 - 120) & $60.0+3.8$ & $58.1+4.09$ & $74.9+3.0$ & $73.0+3.5$ \\
Alanine Amino-Transferase, ALT U/L (12 - 61) & $20.5+2.3$ & $21.5+2.4$ & $16.6+1.6$ & $15.4+1.1$ \\
Aspartate Amino-Transferase, AST U/L (16 - 50) & $27.5+3.2$ & $27.4+3.1$ & $25.4+2.5$ & $25.0+2.6$ \\
Equol (ng/ml) (NA) & $2.1 \pm 0.9$ & $121.8 \pm 13.1$ & $3.5 \pm 1.7$ & $136.7 \pm 9.4$ \\
\hline
\end{tabular}

Normal ranges displayed in parentheses; $\mathrm{NA}=$ not applicable for normal range.

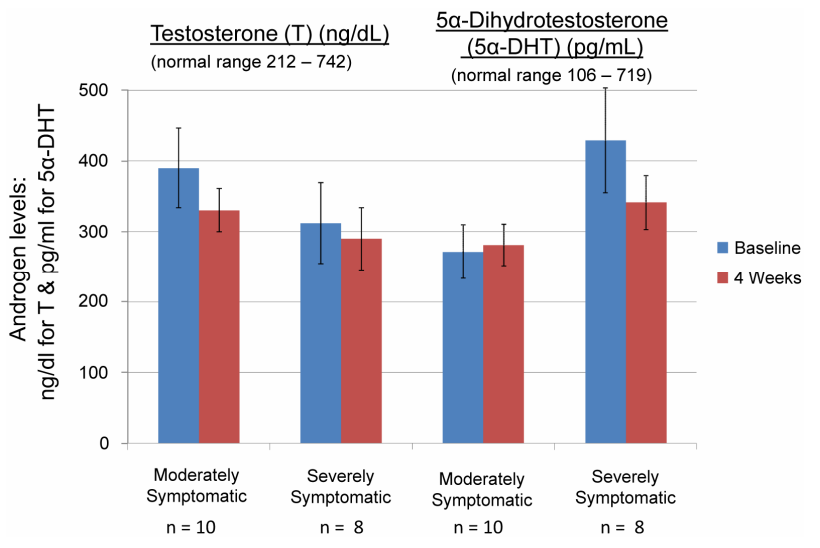

Figure 1. Total testosterone and $5 \alpha$-dihydrotestosterone levels in moderately and severely symptomatic men over the 4 week equol treatment. Testosterone was quantified by chemilum-microparticle immunoassay and $5 \alpha$-DHT by tandem mass spectrometry. There were no significant differences for any of the parameters measured. All values were within normal range.

significantly improved by 4 weeks of equol treatment (Figure 2(b)).

Total IPSS indices as influenced by the equol treatments in the moderately vs. the severely symptomatic groups are shown in Figures 3(a) and (b). In moderately symptomatic men (Figure 3(a)) and in severely symptomatic men (Figure 3(b)) the overall IPSS values significantly decreased from baseline levels by two weeks of treatment and then significantly declined further by four weeks of treatment.

There were no significant changes in quality of life IPSS scores over the four week treatment interval for moderately symptomatic subjects. However, in severely symptomatic subjects for quality of life over the four week treatment interval there was a significant improvement

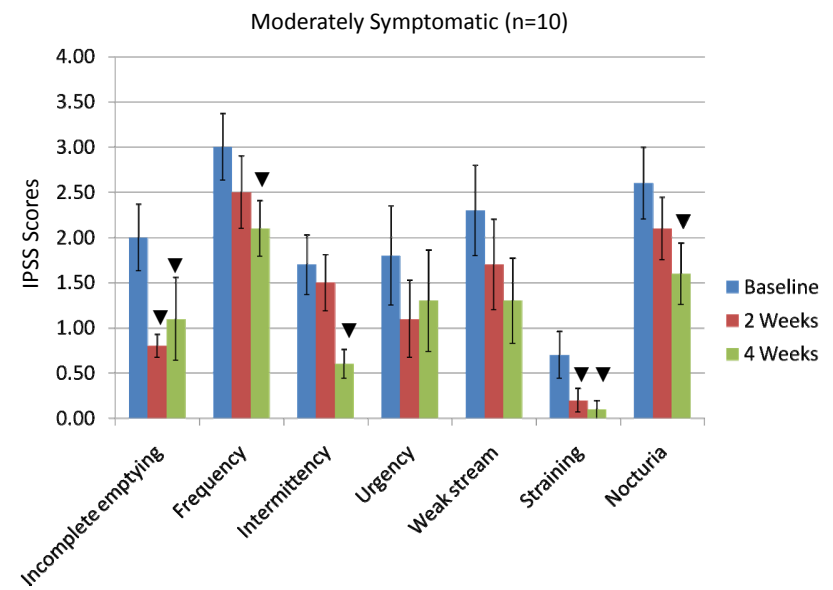

(a)

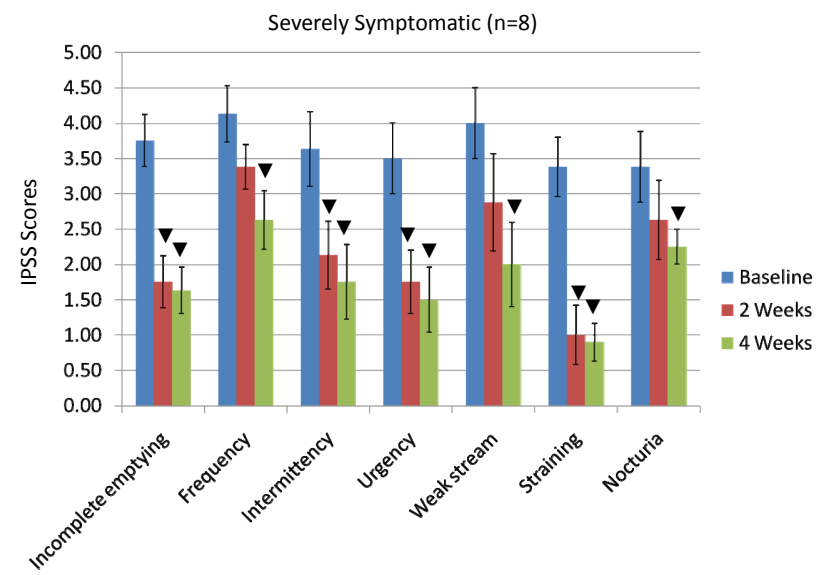

(b)

Figure 2. IPSS values in moderately (a) or severely symptomatic (b) men treated with equol for 4 Weeks; $\nabla=$ significant reduction in IPSS values (within a given parameter) at 2 weeks and/or 4 weeks treatment compared to baseline levels. 


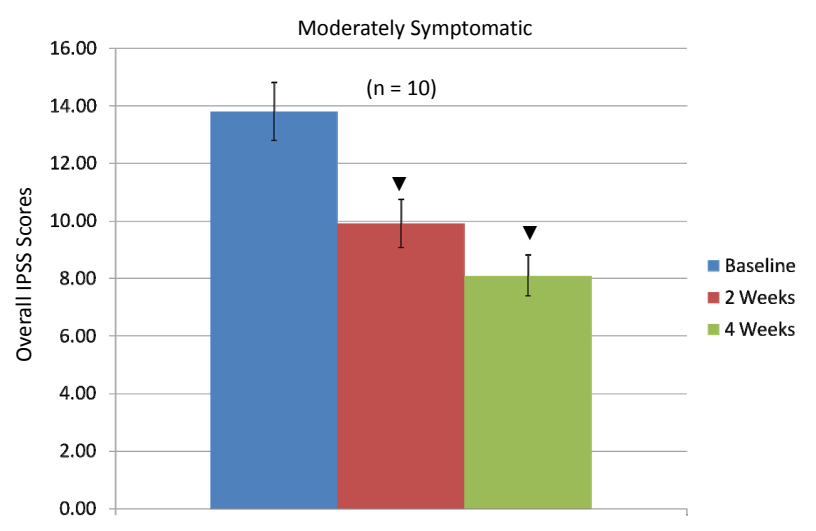

(a)

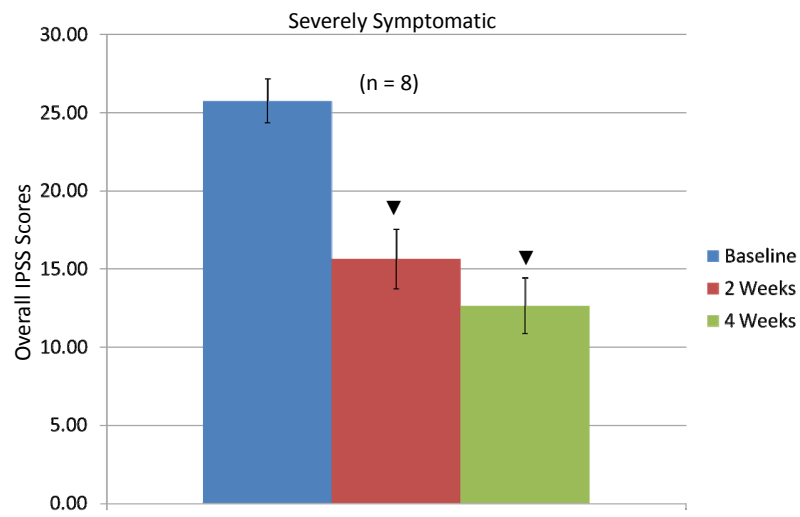

(b)

Figure 3. Overall IPSS values in moderately (a) or severely (b) symptomatic men over 4 weeks treatment with equol; $\nabla$ $=$ significantly decreased IPSS values at 2 weeks and/or 4 weeks compared to baseline levels.

from 4.5 (mostly dissatisfied to unhappy) at baseline to a level of 2.8 toward the mostly satisfied category (quality of life IPSS values not shown graphically). Finally, in the severely symptomatic men total number of urinations (average) per day decreased from 12.1 to 9.2 (or a $24 \%$ decrease) [normal $=4$ to $7 /$ day].

\section{Discussion}

We assessed the safety and efficacy of low dose equol for decreasing symptoms associated with BPH in moderately and severely affected patients. Equol administered at a total daily dose of $12 \mathrm{mg}$ for the 4 week study achieved statistical significance vs baseline values for reducing BPH symptoms, as measured by IPSS parameters. In fact, positive results were observed by 2 weeks. These data compare favorably with previous reports examining $\alpha$-blockers and 5ARIs [2,7] but without the side effects seen in these pharmaceutical treatments.

The potential mechanisms of how equol works are shown in Figure 4. The main mode of action include equol's unique characteristic to bind specifically $5 \alpha$-DHT (and not other steroid molecules or the AR) thereby se-

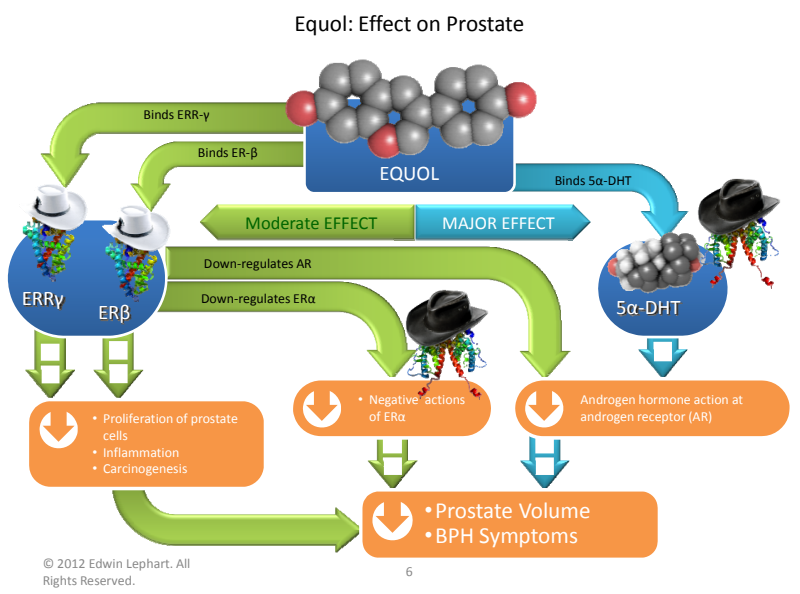

Figure 4. Compounds, receptors and mechanisms of how equol works via various pathways to decrease prostate volume and improve BPH Symptoms in Men. Estrogen Receptor Beta (ER- $\beta)$, estrogen related receptor gamma (ERR- $\gamma$ ), estrogen receptor alpha (ER- $\alpha), 5 \alpha$-dihydrotestosterone $(5 \alpha$ DHT), androgen receptor (AR) and white hats represent positive influences while black hats represent negative influences.

questering it from the AR to decrease this negative androgen impact in the prostate $[24,25]$. The present clinical data confirm previous in vitro and in vivo animal data elucidating this mechanism of action [25].

Conversely, equol also has affinity for ERR- $\gamma$ and ER$\beta$ (to increase positive estrogen-like influences in the prostate). The presence of ERR- $\gamma$ during prostate cancer is indicative of a favorable prognosis, and ERR- $\gamma$ has been shown to slow proliferation in prostate and breast cancer cell lines [3]. Using in vitro cultures examining PC-3 cells, equol has been shown to increase the transcriptional activity of ERR- $\gamma$ [26], thereby enhancing the inhibitory actions of ERR- $\gamma$ on neoplastic growth [3]. The concentrations of equol required to stimulate ERR- $\gamma$ are relatively high but not out of physiological range [26] if equol were applied topically or consumed orally. Also, it is known that equol (particularly S-equol) has relatively high affinity for ER- $\beta$ where it can act as a selective estrogen receptor modulator or SERM $[19,25]$. Previous laboratories, including ours, have shown that isoflavones binding to ER- $\beta$ in the prostate can down regulate the AR and thus decrease negative androgen hormone actions [28]. Moreover, this may explain how isoflavonoid molecules accumulate in prostate tissue and prostatic fluid after oral supplementation [29,30]. Equol's SERMlike binding to ER- $\beta$ within the prostate has positive implications in decreasing proliferation, inflammation and carcinogenesis $[4,25]$ and down regulating ER- $\alpha$ which has a negative impact on prostate health [4]. In part, equol's powerful anti-oxidant and anti-inflammatory activities presumably play an important role in this overall mechanism within the prostate which has been 
demonstrated in human dermal applications [27].

There is evidence that Asian cultures have lower prostate cancer rates compared to Western cultures [9-12,25]. Furthermore, when data is stratified examining individuals that produce equol naturally after soy consumption suggest that lower cancer rates are due to the beneficial influence of this isoflavonoid molecule [31]. Moreover, while equol has been shown to have positive benefits in human prostate health, there is evidence that R-, rather than the S-enantiomer is responsible for the in vivo chemoprotective properties of equol [32]. However, regardless of the actual mechanism by which equol enhances prostate health it is known from other clinical investigations that isoflavone supplementation that increased the production of serum equol in equol-producers resulted in a decline in serum $5 \alpha$-DHT levels in men by approximately $18 \%$ versus before supplementation values [33]. These results are similar to the present findings where a $21 \%$ reduction in $5 \alpha$-DHT was observed in the severely symptomatic group with oral equol treatment with corresponding significant improvements in IPSS values. The reasons moderately symptomatic men did not display any alterations in $5 \alpha$-DHT levels during the intervention are unknown, but the significant positive influence of the equol treatment on IPSS indices over the course of the study may be due to the other mechanisms of actions outlined above.

\section{Conclusion}

Since equol acts directly on $5 \alpha$-DHT and the prostate gland through mechanisms independent of $\alpha$-blockers and 5ARIs, this relatively rapid-acting (within days) therapy may provide an alternative and/or a complementary role for the treatment of BPH. Based upon the positive results of the IPSS indices, without alterations in cardiac or hepatic function parameters and no side effects, equol is likely to be a focus for the relief of BPH symptoms.

\section{Acknowledgements}

This study was supported, in part, by Homeopathic Holdings and TTO/LS College funding \# 19-2215-2011 at Brigham Young University and thanks to Drs: Ronald Rizer (clinic director), Brian Sperber (study physician) and Ms Danielle Wahinekapu (study supervisor) at Stephens Labs, Colorado Springs, Colorado (study site) and Ms Anna White, pre-analytical manager (clinical chemistry) at Utah Valley Regional Medical Center, Provo, Utah for their assistance in this investigation.

\section{REFERENCES}

[1] J. T. Wei, E. A. Calhoun and S. J. Jacobsen, "Benign
Prostatic Hyperplasia," In: M. S. Litwin and C. S. Saigal, Eds., Urologic Disease in America, US Department of Health and Human Services, Public Health Service, National Institutes of Health, National Institute of Diabetes and Digestive and Kidney Diseases, US Government Publishing Office, Washington, 2004, pp. 43-69.

[2] A. Gravas and M. Oelke, "Current Status of $5 \alpha$-Reductase Inhibitors in the Management of Lower Urinary Tract Symptoms and BPH," World Journal of Urology, Vol. 28, No. 1, 2010, pp. 9-15. doi:10.1007/s00345-009-0493-y

[3] T. Fujimura, S. Takahashi, T. Urano, et al., "Differential Expression of Estrogen-Related Receptors Beta and Gamma (ERRbeta and ERRgamma) and Their Clinical Significance in Human Prostate Cancer," Cancer Science, Vol. 101, No. 3, 2010, pp. 646-651. doi:10.1111/j.1349-7006.2009.01451.x

[4] S. J. Ellem and G. P. Risbridger, "The Dual, Opposing Role of Estrogens in the Prostate," Annals New York Academy of Science, Vol. 1155, No. 1, 2009, pp. 174-186. doi:10.1111/j.1749-6632.2009.04360.x

[5] S. J. Berry, D. S. Coffey, P. C. Walsh, et al., "The Development of Human Benign Prostatic Hyperplasia with Age," Journal of Urology, Vol. 132, No. 3, 1984, pp. 474-479.

[6] J. T. Wei, E. Calhoun and S. J. Jacobsen, "Urologic Diseases in America Project: Benign Prostatic Hyperplasia," Journal of Urology, Vol. 179, No. 5, 2008, pp. S75-S80. doi:10.1016/j.juro.2008.03.141

[7] M. Emberton, N. Zinner, M. C. Michel, et al., "Managing the Progression of Lower Urinary Tract Symptoms/Benign Prostatic Hyperplasia: Therapeutic Options for the Man at Risk," British Journal of Urology, Vol. 100, No. 2, 2007, pp. 249-253. doi:10.1111/j.1464-410X.2007.07056.x

[8] M. J. Barry and C. G. Roehrborn, "Benign Prostatic Hyperplasia," British Medical Journal, Vol. 323, No. 7320, 2001, pp. 1042-1046. doi:10.1136/bmj.323.7320.1042

[9] H. Adlercreutz and W. Mazur, "Phyto-Estrogens and Western Diseases," Annuals of Medicine, Vol. 29, No. 2, 1997, pp. 95-120.

[10] K, Griffiths, L. Denis, A. Turkes, et al., "Phytoestrogens and Diseases of the Prostate Gland," Baillieres Clinical Endocrinology Metabolism, Vol. 12, No. 4, 1998, pp. 625-647. doi:10.1016/S0950-351X(98)80008-6

[11] C. Brőssner, K. Petritsch, K. Fink, et al., "Phytoestrogen Tissue Levels in Benign Prostatic Hyperplasia and Prostate Cancer and Their Association with Prostate Diseases," Urology, Vol. 64, No. 4, 2004, pp. 707-711. doi:10.1016/j.urology.2004.04.046

[12] V. U. Kumar, "Phyto-Oestrogens and Prostatic Growth," National Medical Journal of India, Vol. 17, No. 1, 2004, pp. 22-26.

[13] A. Domenica, B. Alessandra, F. Polito, et al., "The Combination of Serenoa Repens, Selenium and Lycopene Is More Effective than Serenoa Repens Alone to Prevent Hormone Dependent Prostatic Growth," Journal of Urology, Vol. 186, No. 4, 2011, pp. 1524-1529. doi:10.1016/i.juro.2011.05.049

[14] G. S. Gerber, "Saw Palmetto for the Treatment of Men 
with Lower Urinary Tract Symptoms," Journal of Urology, Vol. 163, No. 5, 2000, pp. 1408-1412. doi:10.1016/S0022-5347(05)67631-8

[15] A. C. Buck, "Is There a Scientific Basis for the Therapeutic Effects of Serenoa Repens in Benign Prostatic Hyperplasia? Mechanism of Action," Journal of Urology, Vol. 172, No. 5, 2004, pp. 1792-1799. doi:10.1097/01.ju.0000140503.11467.8e

[16] C. Manach, G. Williamson, C. Morand, et al., "Bioavailability and Bioefficacy of Polyphenols in Humans. I Review of 97 Bioavailability Studies," American Journal of Clinical Nutrition, Vol. 81, No. 1, 2005, pp. 230S-242S.

[17] J. M. Smoliga, J. A. Baur and H. A. Hausenblas, "Resveratrol and Health-A Comprehensive Review of Human Clinical Trials," Molecular Nutrition Food Research, Vol. 55, No. 8, 2011, pp. 1129-1141. doi:10.1002/mnfr.201100143

[18] K. D. Setchell and C. Clerici, "Equol: History, Chemistry, and Formation," Journal of Nutrition, Vol. 140, No. 7, 2010, pp. 1355S-1362S. doi:10.3945/jn.109.119776

[19] K. D. R. Setchell, C. Clerici, E. D. Lephart, et al., "SEquol, a Potent Ligand for Estrogen Receptor $\beta$, Is the Exclusive Enantiomeric Form of the Soy Isoflavone Metabolite Produced by Human Intestinal Bacterial Flora," American Journal of Clinical Nutrition, Vol. 81, No. 5, 2005, pp. 1072-1079.

[20] C. L. Frankenfeld, "Dairy Consumption Is a Significant Correlate of Urinary Equol Concentration in a Representative Sample of US Adults," American Journal of Clinical Nutrition, Vol. 93, No. 5, 2011, pp. 1109-1116. doi:10.3945/ajen.111.011825

[21] N. Hounsome, B. Grail, A Tomos, et al., "High-Throughput Antioxidant Profiling in Vegetables by Fourier-Transform Ion Cyclotron Resonance Mass Spectrometry," Functional Plant Science \& Biotechnology, Vol. 4, No. 1, 2010, pp. 1-10.

[22] A. Höjer, S. Alder, S. Purup, et al., "Effects of Feeding Dairy Cows Different-Legume-Grass Silages on Milk Phytoestrogen Concentration," Journal of Dairy Science, Vol. 95, No. 8, 2012, pp. 4526-4540.

[23] E. D. Lephart, "Isoflavones and Prenatal Exposure to Equol," In: V. R. Preedy, Ed., Isoflavones: Chemistry, Analysis, Function and Effects, The Royal Society of Chemistry, Thomas Graham House, Cambridge, 2013, pp. 480-499.

[24] T. D. Lund, D. J. Munson, M. E. Hadly, et al., "Equol Is a Novel Anti-Androgen That Inhibits Prostate Growth and Hormone Feedback," Biology of Reproduction, Vol. 70, No. 4, 2004, pp. 1188-1195. doi:10.1095/biolreprod.103.023713
[25] T. D. Lund, C. Blake, L. Bu, et al., "Equol an Isoflavonoid: Potential for Improved Prostate Health, in Vitro and in Vivo Evidence," Reproductive Biology \& Endocrinology, Vol. 9, No. 4, 2011, pp. 1-9. doi:10.1186/1477-7827-9-4

[26] J. Hirvonen, A. M. Rajalin, G. Wohlfahrt, et al., "Transcriptional Activity of Estrogen-Related Receptor Gamma (ERRgamma) is Stimulated by the Phytoestrogen Equol," Journal of Steriod Biochemistry \& Molecular Biology, Vol. 123, No. 1-2, 2011, pp. 46-57. doi:10.1016/j.jsbmb.2010.11.001

[27] R. Gopaul, H. E. Knaggs and E. D. Lephart, "Biochemical Investigation and Gene Analysis of Equol: A Plant and Soy-Derived Isoflavonoid with Anti-Aging and Antioxidant Properties with Potential Human Skin Applications," Biofactors, Vol. 38, No. 1, 2012, pp. 44-52. doi:10.1002/biof.191

[28] J. M. Hamilton-Reeves, S. A. Rebello, W. Thomas, et al., "Isoflavone-Rich Soy Protein Isolate Suppresses Androgen Receptor Expression without Altering Estrogen Receptor- $\beta$ Expression or Serum Hormonal Profiles in Men at High Risk of Prostate Cancer," Journal of Nutrition, Vol. 137, No. 7, 2007, pp. 1769-1775.

[29] C. D. Gardner, B. Oelrich, J. P. Liu, et al., "Prostatic Soy Isoflavone Concentrations Exceed Serum Levels after Dietary Supplementation," Prostate, Vol. 69, No. 7, 2009, pp. 719-726. doi:10.1002/pros.20922

[30] T. E. Hedlund, P. D. Maroni, P. G. Ferucci, et al., "LongTerm Dietary Habits Affect Soy Isoflavone Metabolism and Accumulation in Prostatic Fluid in Caucasian Men," Journal of Nutrition, Vol. 135, No. 6, 2005, pp. 14001406.

[31] H. Akaza, N. Miyanaga, N. Takashima, et al., "Comparisons of Percent Equol Producers between Prostate Cancer Patients and Controls: Case-Controlled Studies of Isoflavones in Japanese, Korean and American residents," Japan Journal of Clinical Oncology, Vol. 34, No. 2, 2004, pp. 86-89. doi:10.1093/jjco/hyh015

[32] P. J. Magee, M. Raschke, C. Steiner, et al., "Equol: A Comparison of the Effects of the Racemic Compound with That of the Purified S-Enantiomer on the Growth, Invasion, and DNA Integrity of Breast and Prostate Cells in Vitro," Nutrition and Cancer, Vol. 54, No. 2, 2006, pp. 232-242. doi:10.1207/s15327914nc5402_10

[33] M. Tanaka, K. Fujimoto, Y. Chihara, et al., "Isoflavone Supplements Stimulated the Production of Serum Equol and Decreased the Serum Dihydrotestosterone Levels in Healthy Male Volunteers," Prostate Cancer and Prostatic Disease, Vol. 12, No. 3, 2009, pp. 247-252. doi: $10.1038 /$ pcan. 2009.10 